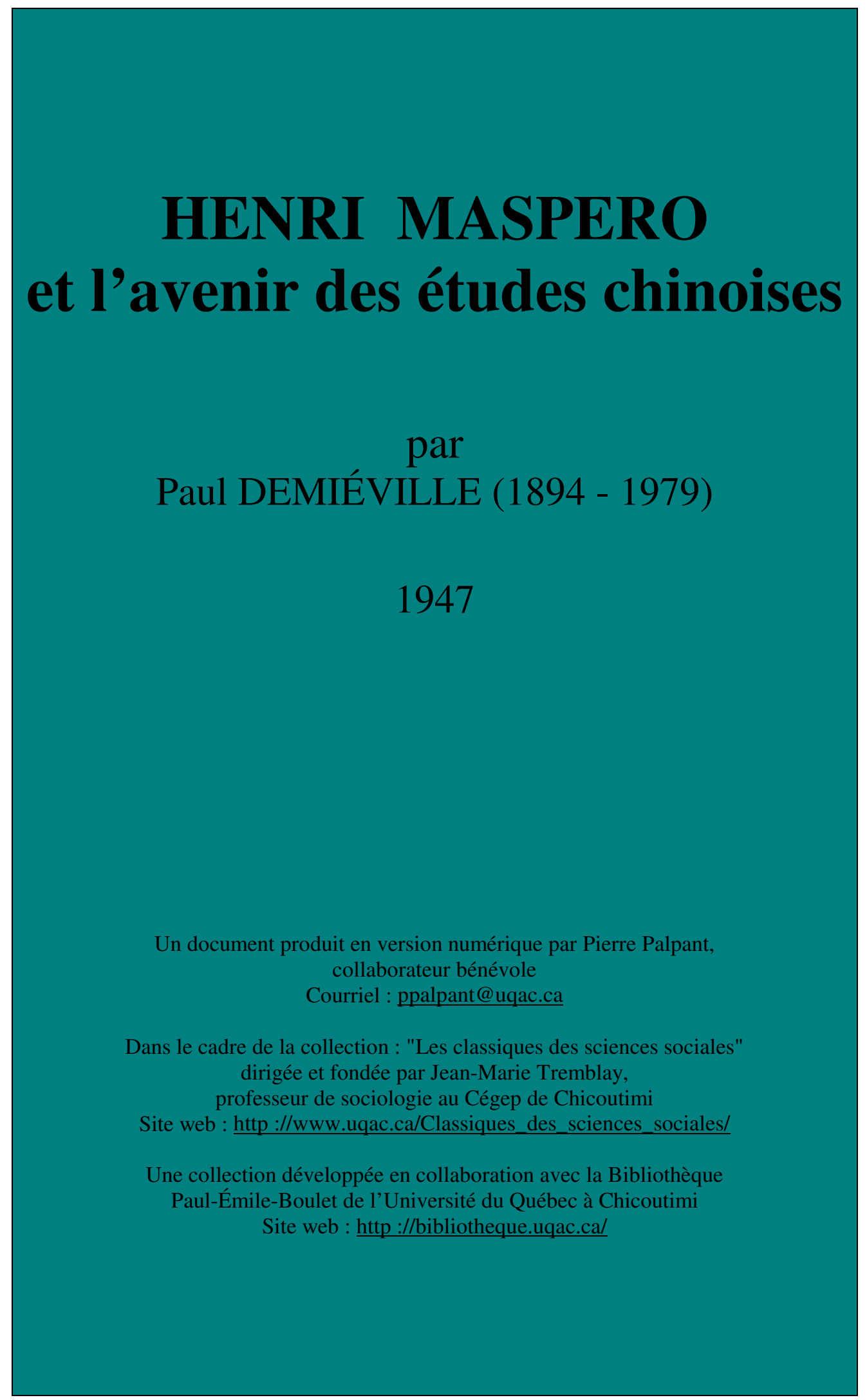


Cette édition électronique a été réalisée par Pierre Palpant, collaborateur bénévole.

Courriel : ppalpant@uqac.ca

à partir de :

\section{HENRI MASPERO et l'avenir des études chinoises}

\section{par Paul DEMIÉVILLE (1894 - 1979)}

T'oung pao, XXXVII, 1947, pages 16-42.

Polices de caractères utilisée : Times, 10 et 12 points.

Mise en page sur papier format LETTRE (US letter), 8.5' x 11'.

Édition complétée le 15 septembre 2005 à Chicoutimi, Québec.

[En publiant un article de Paul Demiéville décédé en 1979, les Classiques ne cherchent évidemment pas à transgresser la réglementation sur les droits d'auteur, mais simplement à faire participer de nombreux lecteurs dans le monde à l'hommage que rend à Henri Maspero son successeur à la chaire de Langue et littérature chinoises du Collège de France.

Peut-être à tort, nous sommes persuadés que nous sommes aussi, par cette diffusion, dans la ligne du profond respect qu'entretenait Paul Demiéville pour la personne et pour l'œuvre d'Henri Maspero.

Si la famille de M. Demiéville ne souhaite pas la diffusion de cet article, qu'elle veuille bien nous le faire savoir : nous retirerons ces pages, en la priant d'accepter toutes nos excuses.] 


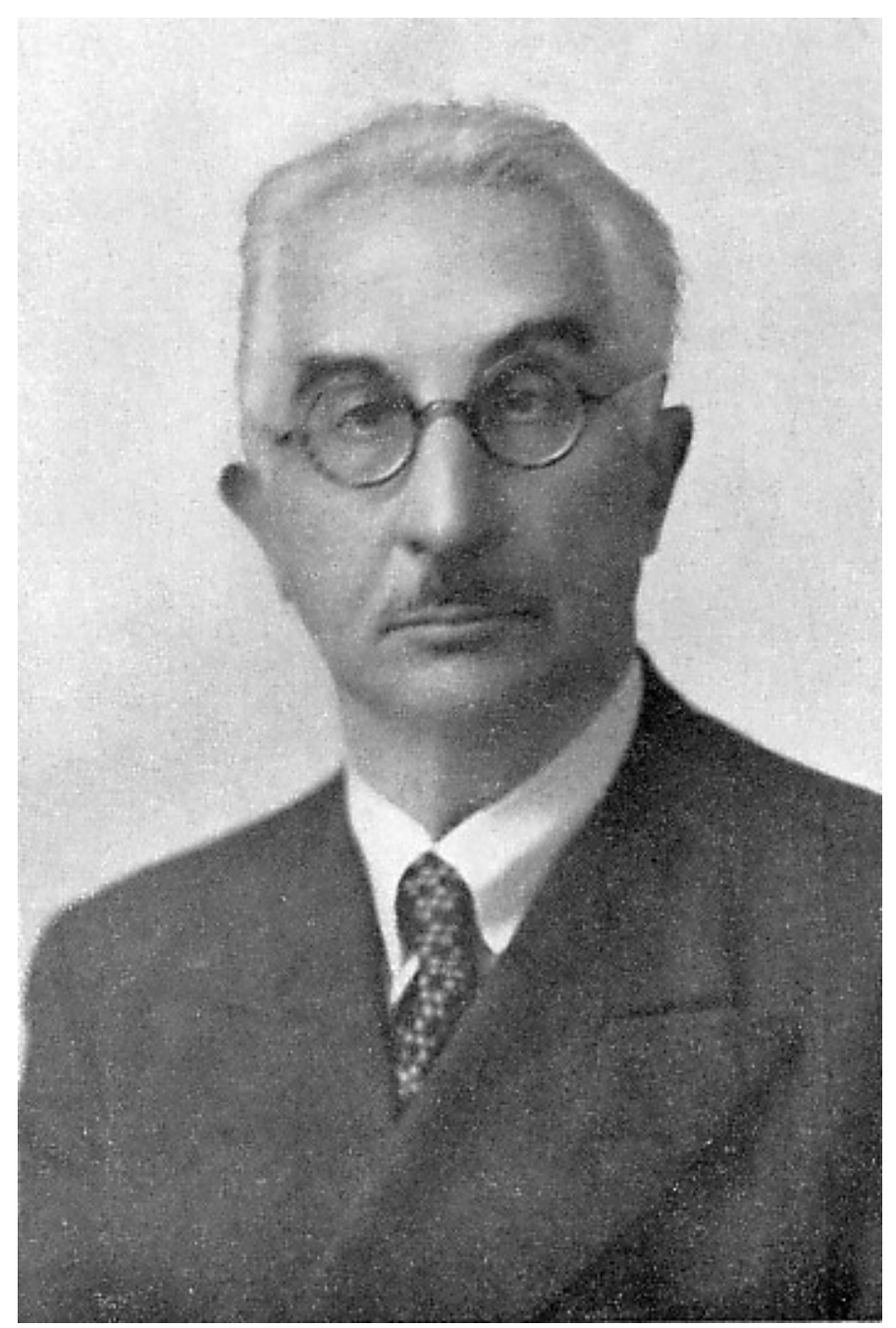

HENRI MASPERO

Membre de l'Institut

Professeur au Collège de France

Né le 15 décembre 1882

Mort au camp de Buchenwald le 17 mars 1945 
En assumant la succession de Henri Maspero, je tiens tout d'abord à exprimer ma profonde gratitude à l'assemblée profes sorale et aux hautes autorités qui ont bien voulu me juger digne d'un si redoutable honneur. Je crois ne mieux pouvoir inaugurer cet enseignement qu'en essayant d'évoquer l'œuvre de l'admirable savant dont un destin tragique a prématurément brisé la carrière. L'entreprise est hasardeuse, et je tremble de manquer à la justesse qui lui était chère. Il y a longtemps quà l'instar de ses illustres prédécesseurs dans cette chaire Henri Maspero s'était placé, par ses travaux comme par son enseignement, à la tête de la sinologie occidentale ; mais il n'avait pas dit tout ce qu'il avait à dire, et la somme de connaissances et d’idées qu’il a emportée avec lui eût sans doute modifié sur plus d'un point l'aspect définitif de s on œuvre : tous les travaux qu'il poursuivait depuis plusieurs années s'orien taient vers de larges synthèses qui devaient en former le couronnement. Cette œuvre inachevée témoigne cependant d'une telle volonté d'ordonnance dirigée, elle est construite avec tant de clarté quỉl ne semble pas interdit d'en dégager les lignes maîtresses. La tentative s'impose d'autant plus que l'œuvre de Henri Maspero est encyclopédique et que la passer en revue, ce sera entreprendre un tour presque complet des études chinoises, dont on pourra faire le point ici et là. De plus, elle est si neuve et d'une telle actualité scientifique quỉl sera permis d'en tirer des éléments de programme et dinndiquer à son propos quelques-unes des perspectives qui s'ouvrent à la sinologie future.

On a dit de Gaston Maspero quil fut le dernier égyptologue à embrasser tout l'ensemble de sa discipline. Sans doute en pourra -t-on dire autant de son fils dans le domaine sinologique; il en avait lui-même le sentiment ; il s'y est du reste épuisé. Il était animé d'une curiosité universelle, un peu à la manière italienne: universelle, mais tournée vers le passé, car son optique resta essentiellement celle d'un historien. De la Chine ancienne, rien ne lui échappa. C'est sur elle que son œuvre est centrée, avec une précision délibérée dont rien ne le fit dévier, sinon les travaux qu'il consacra aux langues et aux religions indochinoises et à l'histoire du peuple d'Annam.

Il n'eut garde, en effet, de se désintéresser d'un pays où il passa treize ans de sa vie et envers lequel la France a des devoirs. L'Annam lui doit l'histoire de sa langue, dont il éclaircit les origines et les affinités, la mise au point critique des principaux tournants de sa tradition historique, et la conservation de ses monuments littéraires et de ses archives épigraphiques et religieuses, méthodiquement recueillis sous son impulsion dans la bibliothèque de l'École française d'Extrême-Orient. Il était d'autre part, trop attentif aux réalités vivantes pour ne pas s'attacher à l'observation et à l'interprétation de celles qu'il avait sous les yeux en Indochine; les livres et les spéculations de cabinet ne lui suffirent jamais. Aussi se mêla-t-il de près aux milieux indigènes, fréquentant presque de jour en jour les fêtes saisonnières ou communales qui rythment et expliquent la vie du paysan tonkinois, dont elles font le charme, et scrutant longuement les coutumes sociales et religieuses des diverses peuplades de l'hinterland indochinois. Il sut, en retour, tirer parti pour ses 
recherches sinologiques des facilités d'enquête qui lui avaient été offertes en Indochine : les matériaux comparatifs qu'il en avait rapportés ne cessèrent de lui servir à élucider des problèmes chinois de tout ordre et de toute époque, depuis la sociologie et la mythologie de l'antiquité jusqu'à la phonologie du Moyen Age et aux religions modernes. Un des titres de gloire de Henri Maspero sera d'avoir introduit dans les études extrême-orientales la méthode comparative, telle que l'Occident l'avait constituée dans le domaine indo-européen. Mais, cette méthode, il la conçut et l'appliqua to ujours en fonction de la Chine; et, depuis son retour du Tonkin, il ne se laissa pas détourner de la pure sinologie au profit de disciplines voisines, comme l'avaient tous fait plus ou moins les maîtres qui l'avaient précédé au Collège de France. C'est la Ch ine même, en son intériorité, mais aussi dans toute son ampleur, qui le retint dès lors exclusivement. Un tel programme lui parut assez vaste ; il le développa en profondeur.

Pas un de ses travaux qui ne représente un progrès de valeur générale, toujours enregistré avec soin en une brève et claire conclusion qui résume les résultats acquis ; pas un domaine de la sinologie où il n'ait marqué sa trace. Et d'abord la linguistique. Celle-ci se présente en Chine dans des conditions particulières, en raison de la nature de l'écriture, qui note le sémantème et non le phonème, ou n'apporte sur ce dernier qu'une information théo rique, algébrique si l'on peut dire, et toujours approximative. Il s'ensuit que, pour faire l'histoire de la langue, il est indispensable d'en établir tout d'abord l'évolution matérielle, c'est -à-dire de restituer les prononciations anciennes et successives des mots. L'étude de la phonétique historique revêt ainsi en Extrême-Orient une importance exceptionnelle; elle nécessite le recours à une technique comparative des plus complexes, les témoignages dont elle dispose étant de nature fort diverse et d'un maniement délicat.

C'est sur le terrain même, à Hanoi, que Maspero se donna une formation de linguiste pour aborder l'histoire phonétique de la langue annamite. Ce travail nécessitait la reconstruction de la prononciation chinoise du $\mathrm{X}^{\mathrm{e}}$ siècle de notre ère, que les Annamites utilisaient à cette date pour la lecture des textes chinois et qui est à l'origine de l'évolution ultérieure du sino-annamite. Maspero se trouva ainsi amené à présenter un tableau systématique de la phonologie chinoise du Moyen Age, qui jusqu'alors n'avait été l'objet que de recherches sporadiques reposant sur des bases comparatives rudimentaires. Ces bases, Maspero les élargit encore dans son ouvrage sur Le dialecte de Tch'ang-ngan, publié à la veille de son retour en France en 1920 et qui, son titre l'indique, porte sur un champ plus nettement délimité : il y est fait état, pour la première fois, de distinctions dialectales dans le chinois ancien ; grâce au double bénéfice d'une documentation plus étendue et d'une mise au point plus serrée, Maspero obtint des résultats qui, joints à ceux d'autres travaux parus à la même époque, peuvent être considérés comme définitifs: les discussions relatives à la phonologie chinoise de l'époque médiévale, à partir du VIIe siècle, ne portent plus que sur des détails. 
Il n'en va pas de même si l'on veut remonter plus haut. Maspero s'était livré à des recherches approfondies sur la prononciation dite archaïque, celle de l'antiquité et des premiers siècles de l'ère chrétienne. Voici vingt -cinq ans qu'il annonçait un travail d'ensemble sur ce sujet : ce travail n'a pas vu le jour, et Maspero accueillit avec réserve les restitutions proposées pour cette période par d'autres spécialistes. L'imprécision des documents proprement chinois ne satisfaisait pas son exigence, et il dut lui sembler prématuré de poursuivre une recherche qui comportait la comparaison avec des matériaux non chinois, alors que les éléments d'une telle comparaison sont encore insuffisamment mis en ouvre.

C'est à Maspero, je l'ai déjà dit, que revient le mérite d'avoir instauré la méthode comparative dans la linguistique de l'Asie orientale : sa Contribution à l'étude du système phonétique des langues thai marque une date à ce point de vue. En remontant à l'ancêtre commun d'une famille dont l'idiome le plus cultivé est le siamois, mais dont il avait pu recueillir dans les régions reculées de l'Indochine française des spéc imens vierges de toute influence extérieure, il indiqua la voie qui permettra peut-être d'é tablir une répartition généalogique des langues de l'Extrême-Orient, pour autant que le type de ces langues ne s'oppose pas à des démonstrations de cet ordre. Les langues thai ont relativement peu divergé les unes des autres; elles se prêtent mieux que d'autres à la comparaison. Celle -ci est beaucoup moins aisée entre les langues dites tibéto-birmanes, autre famille typologiquement parente du chinois. Aux amateurs de rapprochements entre mots chinois et mots tibétains ou birmans, Maspero ne cessa de rappeler quil importait au préalable de restituer le tibétain et le birman anciens, afin de les comparer entre eux et d'aboutir ainsi à restituer un tibéto-birman commun, seul susceptible dêtre, à son tour, rap proché avec fruit du chinois ancien. Or la comparaison interne des langues tibéto-birmanes est à peine amorcée: voilà une tâche urgente pour les chercheurs de demain.

En attendant, Maspero jugea opportun de ne pas pousser à fond l'étude du chinois archaïque, et on ne lui doit, sur cette période de la langue chinoise, qu'un essai traitant, non pas de phonologie proprement dite, mais - le choix est significatif - de ces procédés de dérivation ou, plus exactement, de variation lexicale en vertu desquels les éléments du vocabulaire chinois se groupent en familles de mots. Ces procédés ne constituent pas, même à l'état de vestiges, une flexion grammaticale à la manière indo-européenne; mais si l'importance en a longt emps été méconnue, parce que l'écriture la voilait, elle n'en est pas moins considérable, ne serait-ce que pour l'apprentissage pratique du lexique. Du point de vue théorique, il y a là une sérieuse entorse au principe de linvariabilité des mots chinois, et peut-être un examen systématique de ces variations permettra-t-il de les ramener à des catégories régulières et de reconstituer ainsi, dans la préhistoire du chinois et dans celle des langues voisines où ce phénomène se retrouve un peu mieux conservé, une sorte de morphologie qui donnerait enfin une prise solide à la comparaison généalogique. 
Aussi haut qu'on atteigne le passé de la langue chinoise, celle-ci nous apparaît comme relevant d'un type spécial, répandu dans la plus grande partie de l'Asie ori entale et qui n'a guère changé au cours des siècles. Très simple en son matériel et en ses ressources fonctionnelles, qui laissent transparaître un sous-sol immémorial, mais se prêtant aux élaborations les plus savantes, elle est devenue sous le pinceau d'un grand peuple un incomparable instrument de civilisation, et offre à la linguistique générale une mine de données d'autant plus instructives que ses traits originaux l'opposent fortement aux langues européennes sur la base desquelles s'est édifiée la science moderne du langage. L'analyse typologique et synchronique attira Maspero en même temps qu'il se détournait de l'étude historique des prononciations. Grâce à lui, la sinologie participa à ce mouvement de réaction contre un historicisme trop exclusif, qui se dessine aujourd'hui en linguistique comme dans les autres branches de la science : réaction particulièrement justifiée en Chine, où l'érudition indigène n'a jamais cultivé dans ce domaine, avec l'étymologie et un peu de stylistique, que la phonétique historique. Maspero s'est attaché, à diverses reprises, à scruter le système structural de la langue chinoise, signalant l'influence des moyens d'expression sur les façons de penser et de raisonner, renouvelant aussi, par la comparaison avec d'autres langues du même type, l'explication d'états de langue tels que la koinè parlée moderne, dont on lui doit une description résolument synchronique, mais où il ne se fait cependant pas faute, à l'occasion, de recourir au passé pour éclairer le présent.

Avec sa prescience des problèmes à poser, il avait recueilli dès le début de sa carrière, sur l'ancienne langue parlée, un en semble de matériaux qui reste à peu près tout ce que nous possédons sur l'histoire du chinois vulgaire. C'est par une sorte d'aberration qu'en Chine même aussi bien que dans la sinologie occidentale, les spécialistes semblent s'être donné le mot pour négliger une recherche qui conditionne à plus d'un titre non seulement l'enseignement de la langue, mais aussi la compréhension de ses monuments littéraires : car, ainsi que l'observait Maspero, comment apprécier vraiment la littérature chinoise sans savoir à quel point les gens qui l'ont faite parlaient une langue autre que celle quils écrivaient ? Grâce aux documents dont le dépouillement a été amorcé par Maspero et devra être poursuivi, nous pouvons entrevoir la langue vulgaire du Moyen Age et discerner en quoi elle différait de la langue littéraire. Pour l'antiquité, le départ est infiniment plus délicat à effectuer ; on ne parviendra à reconnaître si les différences grammaticales qui ont été constatées entre les différents textes des Tcheou sont d'ordre stylistique, comme le pensait Maspero, ou d'ordre positivement dialectal comme le veulent d'autres savants, et si la langue de ces textes s'est jamais parlée ou dans quelle mesure elle s'écartait de la langue parlée, que lorsque l'examen systématique des textes sera plus avancé : là aussi les dépouillements nécessaires devront être entrepris.

$\mathrm{Si}$ les questions que pose la dialectologie antique seront sans doute longues à résoudre, il est un domaine où, par contre, les résultats promettent d'être immédiats et d'une abondance à com bler la jeunesse : c'est celui des dialectes contemporains. On en est encore à n'avoir abordé, en sinologie, 
l'étude des parlers locaux, que pour les besoins les plus élémentaires de la phonétique comparée : rien ou presque sur la grammaire, sur le lexique, aucun travail de détail ni sur la géographie linguistique, ni sur les contacts inter-dialectaux, ni sur la gamme très nuancée des divers états de langue et sur leurs rapports très complexes à l'intérieur d'un même parler local. En phonétique même, on a constaté que les prononciations dialectales actuelles, toutes divergentes qu'elles soient, se laissent explique $r$ à partir d'un prototype unique, la prononciation de la métropole impériale telle qu'elle avait é té fixée comme norme officielle au début du VII e siècle après J.-C., lors d'un de ces regains d'unité politique et de centralisation qui survinrent périodiquement au cours de l'histoire de la Chine. On en a conclu que les dialectes modernes eux-mêmes dérivent de la langue parlée à la capitale sous les T’ang, langue qui se serait répandue alors dans toute la Chine et y aurait supplanté les parlers locaux, de même qu'en Grèce les parlers actuels sont issus de la koinè hellénistique et non des parlers antiques. Cette inférence sinspire d'une conception étroitement phonétique des dialectes. Ce qui, dans les dialectes modernes, dérive de la norme commune édictée au VIII siècle, ce n'est en réalité que la prononciation des mots écrits, recueillie sans tenir compte des déviations sémantiques que subissent les mots littéraires dans les parlers locaux, ni des particularités grammaticales propres à ces parlers, ni surtout des prononciations vulgaires coexistant, dans beaucoup d'entre eux, avec les prononciations de lecture et qui seules s'emploient dans la conversation ; il y a même des dialectes où l'on distingue jusqu'à trois prononciations, correspondant à des milieux ou à des usages différents. Cet exemple indique à quel point la dialectologie chinoise est encore déficiente ; il y a là aussi une lacune à combler : mais il sera nécessaire d'enquêter longuement sur place, comme Maspero l'avait fait en Annam.

C'est sur tout à partir de son retour en France, et de son installation dans cette chaire, que Maspero s'adonna à ce qui devait rester dès lors son étude de prédilection, l'histoire de la Chine. Là encore il devait tirer profit des observations qu'il avait re cueillies en Indochine, chez les populations arriérées des régions montagneuses; elles l'aidèrent à recons tituer les phases primitives de la civilisation chinoise, mythes et religions, institutions sociales et conditions économiques. Épris de précision concrète, il se plaisait à ranimer le passé en le confrontant avec ses survivances actuelles : méthode féconde dans cet Extrême-Orient où subsistent côte à côte, comme en une stratigraphie à ciel ouvert, les états successifs d'une culture qui dut être commune, $d$ ans une antiquité reculée, aux Chinois et aux ancêtres de ces populations du Sud-Ouest de la Chine chez lesquelles Maspero en avait retrouvé des débris à peine évolués. Il avait étudié en profondeur, pendant une dizaine d'années, les petites sociétés thai installées aux confins du Tonkin, de l'Annam et du Laos ; il y releva de si suggestives analogies avec la Chine primitive qu'un de ses souhaits, fréquemment exprimé et qu'il mimporte de rapporter ici, était que l'en quête fût étendue aux sociétés du même genre, Lo-lo, Mo-so, Miao-tseu, 
résidant en territoire chinois et moins aisément accessibles à l'investigation occidentale.

Une telle méthode conserve du reste sa valeur, jusque dans l'étude interne de la Chine elle-même, où le conservatisme des institutions et des coutumes fournit à l'historien mainte actualité instructive. Lorsque les incertitudes de la chronologie antique conduisirent Maspero à se faire astronome, pour rectifier, avec sa compétence de philologue, les extrapolations des gens du métier et rétablir dans son contexte historique le développement de l'astronomie chinoise et des instruments matériels qui le conditionnèrent, il commença par les données les plus récentes pour remonter ensuite le cours des siècles. On peut regretter que l'his toire moderne de la Chine ait été abandonnée jusqu ici, en Occident, à des tuteurs pour la plupart dépourvus de formation sinologique ; les sinologues y trouveraient leur profit.

Mais l'histoire à rebours n'était pas le fait de Maspero, et c'est aux origines que dès l'abord il s'attaqua. Il se heurta aussi tôt aux légendes qui forment les débuts de la tradition indigène, à cet ensemble de récits et de thèmes mythologiques que des générations de lettrés nourris de moralisme politique ont réussi, dès une date élevée, à convertir en histoire exemplaire par une des plus remarquables opérations de transposition évhémériste dont l'humanité puisse se targuer : la haute antiquité chinoise en sortit altérée par un gauchissement qui s'est imposé à toute la postérité. La critique en avait été entamée, en France, par Édouard Chavannes ; c'est avec Maspero qu'elle se fit radicale et péremptoire. Pour identifier les mythes derrière la pseudo-histoire, la mythologie thai lui fournit de précieux recoupements positifs : autant la comparaison des langues est ingrate en Extrême-Orient, autant celle des mythologies bénéficie de circonstances extraordinairement favorables. Grâce aux recherches de Maspero et à celles que poursuivait parallèlement, mais sur un autre plan et par d'autres méthodes, ce puissant inventeur que fut Marcel Granet, on peut dire que l'histoire des origines chinoises est aujourd'hui nettoyée de ce que Maspero appelait les fantômes qui l'encombraient.

Ils n'avaient fait tous deux qu'appliquer à la Chine les procédés qui se sont institués chez nous pour l'analyse des antiquités du monde occidental. Ils savaient bien qu'en restant sur leurs positions oc cidentales ils adoptaient une attitude à la fois loyale et, en fin de compte, profitable à la Chine elle-même, où la philologie nouvelle travaille dans le même sens. Aussi Maspero n'a-t-il jamais hésité à soumettre les documents chinois à une critique incisive et sévère, qui domine toute son œuvre et en constitue un des traits caractéristiques. C'est avec une ent ière liberté que cet esprit aigu scruta les secrets d'une civilisation à la fois très fermée et pour tant très humaine, qui ne se livre quà une enquête sans préjugés.

Maspero fut toujours incapable de se payer de mots. Il détestait l'affectation, et rédi geait avec une telle sobriété que la nouveauté de ses vues put échapper parfois à des lecteurs non initiés : comment ceux-ci auraient-ils décelé, derrière telle assertion en apparence banale et la plus naturelle du 
monde, la hardiesse d'une pensée perpét uellement originale ou la richesse d'une documen tation puisée directement aux sources, mais qu'aucune note ne daignait signaler? Sans doute est-ce, pour une part, au commerce des philosophes chinois que Maspero devait cette réserve qui prête tant de distinction à ses écrits et qui faisait le charme de sa personne, mais qui était aussi une défense. Une telle discrétion ne fut pas sans nuire, devant le public, à son livre sur La Chine antique, dans lequel il présenta un exposé synthétique des origines et de l'histoire antique de la Chine. Cet exposé se clôt à la fin du III siècle préchrétien, date cruciale qui marque dans l'histoire de la Chine une rupture de même importance que l'im périalisation, à peu près contemporaine, du monde méditerranéen sous l'égi de de la Macédoine et de Rome. Plus d'une tradition se trouva alors brisée ; ce qui subsista de la littérature antique tomba aux mains d'exégètes nouveaux, qui ne se firent pas faute de retoucher les textes selon l'esprit de leur temps. Aussi le livre de Maspero est-il pourvu, contrairement à sa manière habituelle, d'un commentaire presque perpétuel qui court au bas des pages, procédant à mesure à la critique des sources : au-dessus de cette basse continue s'édifi e la partie constructive de l'ouvrage, riche en harmonies neuves. La Chine antique devait avoir pour suite d'autres volumes consacrés aux premiers siècles de l'époque impériale. La plupart des publications ultérieures de Maspero se rattachent à la préparation de cette suite : vingt ans de déblaiement pour mettre sur pied un précis de quelques siècles d'histoire de la Chine, jusqu'au V Ie de notre ère : voilà une leçon pour les généralisateurs, et un motif d'amer regret pour nous qui nous trouvons frustrés d'un tel ins trument de travail.

Ses recherches sur le haut Moyen Age chinois ne l'empêchèrent pas de continuer à s’intéresser à la période antique ; il y revint sans cesse, soucieux de compléter ou de rectifier ses travaux antérieurs à la lumière des découvertes nouvelles, exploitant en particulier deux disciplines qui, lors de la publication de La Chine antique, n'avaient pas encore reçu le développement qu'elles ont pris depuis lors en ce qui concerne la haute antiquité : je veux parler de l'archéologie et de l'épigraphie.

La fouille archéologique est toute récente en Chine. Le respect des tombes ancestrales et les croyances qui s'attachent au sol, considéré comme sacré, se sont longtemps opposés à ce qu'on violât les gisements souterrains ; seuls y trouvaient leur compte les pillards. Il a fallu la découverte, il y a une vingtaine d'années, du Pithé canthrope de Pékin, puis celle d'un site de la dynastie des Chang, datant de la fin du II ${ }^{\mathrm{e}}$ millénaire avant l'ère chrétienne, pour que les champs de fouille s'ouvrissent officiellement en divers points de la Chine septentrionale. Maspero suivit attentivement les travaux des archéologues chinois, sans y participer lui-même. Ses contributions à l'archéologie sont d'un autre ordre : elles portent la marque de l'historien. Il avait entrepris, juste avant la guerre de 1914, la prospection d'une région du Tchö-kiang où les vestiges du passé n’ont guère été épargnés par l’insurrection des T’a i-ping, cet épisode si curieux de l'histoire moderne de la Chine, qui mériterait d'être étudié à fond comme type de mouvement populaire et religieux et comme prodrome de l'ère actuelle. De ce champ dévasté, Maspero réussit à tirer une 
moisson archéologique appréciable, grâce à l'utilisation exhaustive des sources d'information indigènes et notamment de ces monographies locales qui sont en Chine le vademecum du voyageur et mériteraient d'être imitées ailleurs. Plus tard, c'est à la lumière d'objets parvenus dans les collection européennes ou reproduits dans des publications d'Extrême-Orient qu'il s'attacha à interpréter les textes relatifs à la vie matérielle de l'époque des Han et reconstitua, en particulier, le type de la maison antique. La tendance qui le portait à serrer le concret de plus en plus près ne cessa de s'accuser jusqu'à la fin de sa carrière, et on le vit, au cours de la dernière communication qu'il fit à la Société Asiatique, partir de remarques sur le labour à la houe, tel qu'on le pratiqua dans la Chine antique, pour s'élever aux plus hautes considérations d'ordre social et littéraire.

Mais c'est surto ut l'épigraphie antique qui devait retenir son atten tion au cours de ses dernières années. Il foulait là une piste à peu près vierge pour la sinologie occidentale. Les fouilles récentes ont fait remonter de plusieurs siècles la paléographie chinoise, en fournissant des textes gravés sur os ou sur écaille à l'époque des Chang, dans la deuxième moitié du deuxième millénaire avant notre ère ; elles ont aussi facilité ou activé le travail d'authentification, de classement chronologique et de déchiffrement des inscriptions sur bronze des Tcheou, qui couvrent la plus grande partie du dernier millénaire préchrétien. C'est à c elles-ci que Maspero recourut pour en tirer des données relatives à l'histoire judiciaire et économique de la dynastie des Tcheou, plus précisément aux contrats illustrant la procédure civile et le régime de la propriété foncière: matières sur lesquelles la littérature traditionnelle, si pauvre en realia, n'a presque rien à nous apprendre. L'histoire a été considérée en Chine comme un 'miroir' d'édification tendu à la postérité : aussi n'a-t-on jamais tenu dans ce pays, où l'on se montre pourtant si respectueux de tout ce qui est écrit, à la conservation des documents d'un genre jugé dépourvu de valeur instructive. Aggravée par une grande découverte chinoise, celle de l'imprimerie qui, dès le début du présent millénaire, supplanta totalement la tradition manuscrite, une telle attitude n'était pas favorable au développement d'une con ception scientifique de l'histoire : aussi la chasse au document direct doit-elle user en Chine de maints détours pour débucher un gibier élusif. C'est ce qui fait l’importance des archives manuscrites découvertes, au cours du dernier demi-siècle, soit à Touen-houang par Paul Pelliot et d'autres, soit dans les sables de l'Asie Centrale d'où Aurel Stein tira ces fiches vénérables au déchiffrement desquelles Maspero consacra, après Chavannes, des années de travail. Les manuscrits d'Asie Centrale se rapportent aux premiers siècles de notre ère, ceux de Touen-houang descendent jusqu'à l'an 1000 ; le contenu en est assez varié. Pour la période antique, les inscriptions offrent moins de ressources : oracles divinatoires, consécrations rituelles, actes juridiques ou administratifs, c'est tout ce qu'elles nous livrent pour compléter les rares informations d'ordre positif qu'une enquête attentive parvient à dépister dans les textes classiques. Maspero possédait une formation juridique ; il s'était fait la main, dès ses débuts, à ce genre de recherches, en retraçant au cours d'un séjour en 
Égypte l'organisation financière et fiscale de l'empire des Ptolémées. Il sut extraire des inscriptions antiques les éléments d'un tableau du régime féodal chinois, envisagé du point de vue économique; dans un travail ultérieur, il esquissa en raccourci l'évolution des modes de la propriété foncière en Chine depuis les origines jusqu'à nos jours ; et une préface qu'on lui demanda pour un ouvrage d'histoire moderne lui fournit l'occasion d'insister à nouveau sur l'importance des facteurs éco nomiques, trop négligés dans cet ouvrage, en expliquant par eux une de ces mutations dynastiques qui scandent l'histoire politique de la Chine, la chute, au XVII siècle, de la maison des Ming et l'avènement des Mandchous. Ces travaux sont presque uniques en leur genre dans la sinologie occidentale. Même en Extrême-Orient, c'est surtout à l'examen des faits contemporains que se sont jusqu'ici con sacrés les jeunes économistes formés à l'école de l'Amérique, ainsi que leurs maîtres américains, auxquels la sinologie doit dans ce domaine tant de remarquables contributions : là aussi, il faudra tenir compte du passé.

Le progrès de l'archéologie à la faveur de fouilles contrôlées, la mise en auvre des inscriptions antiques et, pour le Moyen Age, celle des manu scrits de l'Asie Centrale et de Touen-houang, sinscrivent au programme de la sinologie de demain. C'est du reste sur ces trois points que portait déjà principalement l'effort de la brillante école d'érudits chinois dont les travaux se développaient avec succès lorsque la tragédie nationale vint temporairement les suspendre : il faut espérer que cet effort pourra désormais se poursuivre en Chine dans des conditions propices, et que la science occidentale y prendra une part active; Maspero a ouvert la voie.

Cette intelligence si ouverte avait trop le sens des grands problèmes humains pour que, dans le portrait quil nous a laissé de la Chine, les traits religieux ne se trouvent pas fortement accusés. On a dit que les Chinois n'avaient pas de religion : toute l'œuvre d e Maspero apporte des démentis à cette assertion. Peut-être celle-ci repose-t-elle sur une conception trop étroite de ce quill faut entendre par religion. Depuis la Querelle des Rites, on discute en Europe sur les définitions qui peuvent être données de ce terme dans ses applications à la Chine : peut être des solutions ne sont-elles possibles que sur un plan proprement religieux. C'est sur un autre plan, cela va de soi, que Maspero se plaçait en étudiant les religions de la Chine ; mais il avait un sentiment trop fin et trop lucide des valeurs religieuses (ne comptait-il pas Benjamin Constant parmi ses ascendants maternels ?) pour ne pas s'attacher à pénétrer par l'intérieur les attitudes diverses que les Chinois ont adoptées, soit successivement, soit simultanément, devant ces questions vitales.

La religion de l'antiquité chinoise, dont sa vision fut vivifiée par les observations qu'il avait pu faire en Indochine, lui ap parut comme un système à la fois clos et collectif, centré sur la famille et sur le groupe territorial, sur le culte des ancêtres et sur celui du sol, assise divinisée de la communauté humaine et gîte de ses morts. C'est là que Maspero voyait le noyau de la religiosité chinoise, demeuré toujours intact sous les éléments différents qui 
vinrent s'y agréger peu à peu : car en Chine, contrairement à ce qui s'est passé chez nous, le paganisme de l'antiquité n'a pas été étouffé par les nouvelles tendances, de caractère en même temps universaliste et individualiste, qui devaient s'y superposer au cours des siècles.

C'est à l'époque dite des Principautés Combattantes, dans la convulsion qui annonça, au IVe et au III ${ }^{e}$ siècle avant notre ère, l'effondrement du vieux fédéralisme féodal, que ces tendances commencèrent à s'affirmer : époque d’intense fermentation religieuse et philosophique, où fleurirent des écoles et des maîtres voués aux libres controverses et qui nous a légué quelques-unes des plus grandes œuvres de la littérature chinoise. Alors s'épanouit notamment une école de gnose mystique quillustrèrent de prestigieux écrivains dont devait se réclamer plus tard la secte taoïste. Dans une étude consacrée à Lao-tseu et à Tchouang-tseu, Maspero assigna à ces deux penseurs la place qui leur revient, au premier rang de la mystique universelle, dont il souligna à ce propos l'étonnante unité. A ceux qui cherchent en Chine, dans cette civilisation pour nous la plus lointaine et longtemps la plus isolée, des gages d'identité et de communauté entre les hommes, il n'est pas, en effet, d'é tude plus rémunératrice que celle de la mystique; à feuilleter Plotin ou Fénelon au sortir de certains textes chinois, on a parfois la sensation de voir se superposer aux mots grecs ou français les caractères chinois qu'on vient de lire.

La veine mystique devait toujours rester vivace en Chine, avec sur le plan social et politique une certaine tendance à l'anarchie libertaire et volontiers violente. Mais, dans l'empire totalitaire fondé par les Ts’in et les Han, cette veine disparut dans des couches souterraines d'où elle ne devait resurgir au plein jour de l'histoire et de la littérature qu'après une période assez prolongée. Jusque vers l'an 200 de notre ère, sous tout le règne des Han, ces Romains de l'Asie, ce fut la doctrine des lettrés, ce que nous appelons le confucianisme, qui s'érigea en orthodoxie et fut imposée en surface par un système rigide d'enseignement officiel ; de là date ce mandarinisme à base d'examens dont les conséquences, bonnes ou mauvaises, devaient commander tout l'avenir politiqu e et linguistique de la Chine.

Abordant l'étude de cette époque pour faire suite à sa Chine antique, Maspero rencontra des difficultés d'un genre nouveau. Si, à partir des Han, les documents se font plus nombreux et moins sujets à caution, l'exploration scientifique en était, par contre, beaucoup moins avancée, et les grandes perspectives elles-mêmes restaient à tracer. Mais c'est surtout en matière de religion que Maspero se trouva devant une sorte de vide qui dut paraître intolérable à un savant si pénétré de l'importance des facteurs religieux dans le développement des sociétés humaines. Il se rendit compte alors de la valeur capitale d'une source d'information qu'on avait né gligée avant lui : c'est dans les commentaires des livres classiques, constata-t-il, que s'étaient exprimées sous les Han les doctrines religieuses et philosophiques du confucianisme. De même que la pensée chrétienne peut être considérée comme une exégèse des Écritures, ainsi en Chine, mais de façon plus littérale encore, et non seulement 
sous les Han, et en dehors aussi du confucianisme, c'est sous la forme d'interprétations de la tradition, et, matériellement, de gloses accrochées de façon plus ou moins arbitraire aux textes canoniques, que se présente dans une large mesure l'évolut ion vivante et créatrice des idées et des croyances.

Maspero en donna un exemple en étudiant la première histoire de l'antiquité chinoise qui nous soit parvenue, le Tso tchouan ou Tradition de Tso. Ainsi que l’indique son titre, cet ouvrage prétend repré senter l'exégèse traditionnelle qui avait cours dans l'une des écoles où se transmettait et s'expliquait un des textes placés sous l'autorité de Confucius, les Printemps et Automnes (Tchouen ts'ieou), annales de la seigneurie de Lou dont le saint était originaire. Par une analyse des sources de la Tradition de Tso, Maspero montra comment ce livre, qui finit par passer lui-même au rang d'autorité scripturaire, ne se rattache en fait que de façon partielle et artificielle aux Printemps et Automnes et comment, d'autre part, les virulentes discussions qui n'ont cessé de sévir en Chine, depuis une dizaine de siècles, autour de son authenticité et de ses origines, s'inspiraient non pas d'une recherche objective, d'ordre historique ou philologique, mais de préoccupations proprement religieuses. Ainsi envisagé, le confucianisme ancien reprend vie à nos yeux et les commentaires des classiques, considérés non plus dans ce quils peuvent nous apprendre sur les classiques eux-mêmes, mais en eux-mêmes et comme témoins des conceptions de leurs auteurs et de leur temps, nous apparaissent comme des documents de premier ordre qui devront désormais retenir l'attention des historiens de la pensée chinoise. Les classiques eux -mêmes ne gagneront-ils pas à être ainsi dissociés de la tradition ?

C'est surtout sur une autre des religions de la Chine, le taoïsme, que Maspero fit auvre éminemment originale. On sait comment la doctrine du tao, chemin de vie élevé à la hauteur d’un principe métaphysique, donna son nom dès les premiers siècles de notre ère à une secte qui, sous l’impulsion du bouddhisme, s'organisa en église, avec un clergé, un culte, des rites, et surtout des pratiques d'ordre à la fois physique et psychique dont le but était d'assurer une immortalité conçue de la façon la plus réaliste comme une survivance du corps matériel. Il y avait là un complexe bien chinois de bon sens crûment terre-à-terre, d'aspiration naïve au salut, d'irrévérence à la fois ironique et prudente à l'égard d'un panthéon de divinités calqué sur le m andarinat de ce monde, enfin de techniques immémoriales où l'on ne sait ce qui l'emporte, de l'ingé niosité empirique ou de l'absurdité des théories : tout cela représentant, selon Maspero, « la solution spécifiquement chinoise des grands problèmes de morale et de métaphysique religieuse qui, par tous pays, ont agité l'esprit des hommes quand les vieilles religions sociales primitives eurent cessé de leur suffire, et qüils se sentirent le besoin de religions personnelles ». La grande époque du taoïsme se situe dans le haut Moyen Age, entre le III e et le VI siècle de notre ère; il fut bientôt battu en brèche par le bouddhisme, dans lequel il finit par se résorber, non sans l'avoir, au préalable, imprégné en retour de son influence indélébile. La décadence du taoïsme est aujourd'hui 
fort avancée, et lorsque Maspero s'aventura dans la masse indigeste des Écritures taoïques, devenues rarissimes dans leurs éditions anciennes, mais récemment réimprimées en Chine, il dut procéder à tout un déchiffrement terminologique de ces textes dont le lexique technique n'est plus compris. De ce long et rebutant travail, il n'avait tiré jusqu'ici que la matière de trop brefs articles. On pouvait attendre de lui une véritable résurrection de cette religion, une des plus curieuses du monde, et combien révélatrice de la mentalité chinoise ! Maspero était le seul homme vivant, aussi bien en Asie qu'en Europe, qui en eût exploré méthodiquement l'histoire et les dessous techniques, au prix d'un effort poursuivi avec opiniâtreté pen dant un quart de siècle : il n'est presque pas d'année, depuis 1921, où l'un au moins de ses cours dans cette chaire n'ait porté sur le taoïsme. Il disparaît au moment où une maturité féconde eût eu raison des énigmes et des scrupules qui l'avaient retenu de publier les résultats de ses recherches. Voilà ce que la science a perdu à Buchenwald.

Depuis son introduction en Chine vers le début de l'ère chrétienne, le bouddhisme a été lié de si près au taoïsme que Maspero ne pouvait s'en désintéresser. La sinologie européenne s'est long temps mise, dans l'étude du bouddhisme, au service de l'indianisme, lui fournissant docilement, sur cette religion que l'Inde a reniée après l'avoir donnée à l'univers, les informations qui peuvent être tirées des traductions chinoises de textes sanskrits perdus, ou des témoignages pieusement recueillis aux lieux saints par les pèlerins chinois ; et Maspero pouvait écrire, il y a quelques années, que tout reste à faire sur le bouddhisme chinois. Il entendait par là cette forme proprement chinoise qu'a revêtue le bouddhisme au cours de sa diffusion en Chine, les développements quill y a reçus, le rôle quiil a joué dans l'histoire de la pensée et de la culture chinoises. Imagine-t-on une histoire de la religion chrétienne où il ne serait tenu compte des sources latines qu'en fonction du chris tianisme hellénistique, voire même judaïque ? L'exemple est forcé : il le faut tel pour faire sentir l'insuffisance des exposés du boud dhisme jusqu'ici présentés au public d'Occident.

Sur les origines du bouddhisme chinois, Maspero s'acquitta tout d'abord de sa besogne habituelle d'e xécuteur de fantômes, rendant à la légende les traditions admises. Puis, passant aux données positives, il put montrer par des témoignages précis, recueillis au cours de ses recherches sur le taoïsme, comment ce fut dans des milieux taoïstes que le bouddhisme simplanta pour la première fois. Il y est attesté avant la fin du $1^{\text {er }}$ siècle de notre ère, et pendant plusieurs siècles ce devait être presque exclusivement dans ces milieux que se recrutèrent en Chine les adeptes de la Vraie Loi. C'est qu'à plus d'un égard le taoïsme avait préparé au bouddhisme un terrain congénère : même aspiration au salut personnel conçu comme une libération de la personne ; même rôle fondamental de la mystique, cette mystique étant de la même espèce impersonnelle et liée, en Chine comme en Inde, à des techniques d'ordre physiologique; même superstructure d'une métaphysique assoiffée 
d'absolu et qui, dans le bouddhisme du Grand Véhicule, a vait eu raison du relativisme du Petit Véhicule; et enfin même propos de ne pas tuer le relatif par l'absolu, mais au contraire de les vivifier l'un pa $r$ l'autre : d'où un souci constant de neutraliser les antithèses. Toutefois, derrière ces traits qui apparentent spirituellement la Chine et l'Inde et les opposent, sur le plan religieux, à l'Occident sémitique et européen, les incompatibilités ne manquaient pas. Pour les Chinois, la vie prime tout : la réalité suprême est, à leurs yeux, mutation créatrice ; et leur sensibilité était constitutionnellement trop vibrante au concret pour qu'ils pussent se plier au quiétisme indien et à la sombre dialectique du bouddhisme. Aussi la réaction ne tarda-t-elle pas. Mais il y avait assez de points communs pour qu'entr e le bouddhisme et le taoïsme s'établît une sorte d'osmose qui, dans le vocabulaire technique en particulier, rend souvent difficile le départ entre ce qui provient de l'un et ce qui est emprunté à l'autre.

C'est ce qu'on peut observer notamment dans les écrits des remarquables écoles philosophiques qui se développèrent sous les Trois Royaumes et les Six Dynasties, entre le III et le $\mathrm{VI}^{\mathrm{e}}$ siècle de notre ère, époque turbulente et féconde où devait germer plus d'une doctrine appelée à un grand avenir. Dès lors se manifeste ce goût du compromis et du syncrétisme qui détermina l'orientation de la pensée chinoise en ses phases ultérieures, de même quil explique la continuité des institutions politiques chinoises. Lorsque avec le bouddhisme elle se trouva en face d'une autre grande civilisation, cette civilisation chinoise qu'on a dite hermétique et figée allait se montrer capable d'un effort d'assimilation sans parallèle dans l'his toire du monde. La civilisation antique, en Occident, n'a pas sur vécu au christianisme ; en Chine, c'est le bouddhisme qui a succombé, mais dans des conditions toutes particulières. À l'époque des T'ang, du VI $\mathrm{I}^{\mathrm{e}}$ au $\mathrm{IX}^{\mathrm{e}}$ siècle, le bouddhisme domine toute la production philosophique chinoise ; et lorsqu'on parcourt les travaux de Hiuan-tsang, ce génie plus connu en Europe comme voyageur et comme historien que sous son aspect de traducteur et d'exégète des grands traités de la scolastique sanskrite, on demeure confondu de l'im mensité de son œuvre, de la rapidité avec laquelle elle fut réalisée, de l'exactitude et de la pénétration dont elle fait preuve dans l'interprétation de la plus abstraite des langues indo-européennes et des systèmes de pensée les plus subtils auxquels l'Inde, mère des dogmes et des mystères, ait donné naissance en sa gloire. Si la Chine a su prendre alors au monde extérieur ce qu'il offrait sans doute, à cette époque, de plus brillant dans l'ordre de la pensée, comment donner raison à ceux qui, de nos jours, la jugent incapable de s'assimiler les sciences et les techniques de l'Occident ? Et si l'on prétend que l'esprit scientifique lui a manqué dans le passé, on peut répondre qu'avant Hiuan -tsang la spéculation abstraite n'avait pas non plus été le fort des philosophes chinois.

Mais c'est en les adaptant à ses besoins et à ses aptitudes qu'elle s'appropriera les principes utiles de la civilisation moderne, comme elle le fait déjà avec tant d'énergie et d'efficacité. La Chine est lente à absorber ses emprunts ; l'absorption est profonde et totale. A la fin de l'époq ue des T'ang, le confucianisme lui-même avait irrémédiablement subi l’influence du 
bouddhisme; il est vrai qu’il se défendait d'être touché : n'est-ce pas justement qu'il l'était ? Et ce qu'on appelle le né o-confucianisme des Song, l'école de Tchou Hi, qu i se constitua à partir du XIe siècle et devait donner à toute l'époque moderne son orthodoxie officielle, nous apparaît de plus en plus comme une synthèse doctrinale où l'apport bouddhique, transparent dans la terminologie, joue un rôle d'autant plus important qu'il est moins avoué : «C'est la profondeur même de l'influence bouddhique qui la masque », écrivait Maspero. Ces délicates réactions de défense sont familières à l'Extrême-Orient; il en faut saisir le jeu souple et parfois déroutant. Si le bouddhisme et le taoïsme semblent, aux temps modernes, être effectivement devenus improductifs en Chine, c'est qu'à partir de Tchou Hi le confucianisme en avait assimilé les éléments vivants au point de les entraîner dans son évolution sans qu'on eût plus cons cience de leur hétérogénéité. Aussi Maspero voyait-il avec raison dans la religion populaire actuelle, sur laquelle il s'est penché avec tant de compréhen sion, soit au cours de ses séjours dans l'intérieu r de la Chine, notamment au Tchö-kiang et au Kiang-sou, soit à la fin de l'autre guerre lorsque ses fonctions militaires le mirent en contact avec des travailleurs chinois, non pas une juxtaposition absurde et inhumaine des «trois religions » de la Chine, confucianisme, taoïsme et bouddhisme, mais un complexe vivant issu de leur fusion et constituant une religion nouvelle : nouvelle, et néanmoins restée si proche du vieux fonds de croyances, de rites et de recettes qui dans la nuit des temps avait précédé les «trois religions ».

Ainsi s'achève cet es sai de commémoration d'une auvre si étendue et si diverse qu'elle nous aura fait parcourir, comme je vous en prévenais, presque tous les registres de la sinologie. Il n'est pas jusqu'à la littérature proprement dite qui n'ait attiré l'attention de Maspero. Les belles-lettres chinoises ne le séduisaient guère; il était rebuté par les raffinements d'une rhétorique qui transfigure la langue jusqu'aux limites de l'artificialité. Il en laisse cependant un tableau d'autant plus neuf que ce vaste sujet est resté jusqu'ici frappé, en Occident, d'une sorte d'ostracisme qui s'explique, sîl ne se justifie, par l'extrême difficulté des textes, très disparates sous l'apparente uniformité de la langue, et par l'ingratitude des traductions; il faudra bien pourtant qu'on s'engage dans cette voi e épineuse. Maspero n'était pas philosophe ; mais on lui doit l'étude la plus fouillée que nous ayons sur la logique chinoise. Il préparait aussi une histoire de l'art, où la rigueur de sa critique aurait trouvé à s'exercer de façon particulièrement salutaire, une histoire des institutions, proche de son achèvement; d'autres travaux en cours, des notes et des dossiers sans nombre attendaient son retour. . .

Hélas ! ce n'est pas impunément qu'un esprit de cette trempe avait souten u le long effort de se colleter ainsi avec toute une civilisation. Il s'était livré à des lectures immenses, telles que jamais Européen n'en avait entrepris. Que de nuits blanches jusqu'au delà de l'aube ! S'il n'était pas un bénisseur, c'est qu'il était aussi sans indulgence pour lui-même, Par surcroît, jamais il n'esquivait un devoir social ni aucune des obligations que lui imposait sa 
situation académique. Il avait trop fréquenté Confucius pour méconnaître la sagesse des rites, et les taoïstes lui avaient appris, comme dit Tchouang-tseu, que « pour vivre caché il faut commencer par ne pas se cacher ». Il prodiguait son temps à ses élèves, à ses confrères, à ses amis d'Occident et d'Orient. Une énergie rebelle et le démon de la recherche, qui lui faisait oublier rendez-vous et le reste quand sa curiosité scientifique était en branle, avaient toujours soutenu sa constitution d'ailleurs robuste, mais qu'il avait soumise à un rude régime. Cependant il restait sujet aux récurrences d'une fièvre maligne, contractée naguère dans la brousse annamite, et les événements du début de cette guerre avaient profondément frappé en lui le patriote. Aussi fut-ce un homme las, usé par un travail d'une excessive intensité, que les Allemands jetèrent en prison et traînèrent à Buchenwald. Il y subit des traitements qui eurent raison, non pas de sa résistance morale, mais des ressources physiques que lui avait laissées une vie dévouée à la science. Si Henri Maspero est mort pour la France, il est mort aussi au service de la science.

Précédée de la mort prématurée de Marcel Granet, suivie, à six mois de distance, de la mort de Paul Pelliot, la mort de Henri Maspero laisse dans la sinologie occidentale un vide irréparable. Je crois être fidèle à la mémoire de ces trois savants, victimes directes ou indirectes du drame de notre temps, en rendant hommage ici à celle de leur maître commun, Édouard Chavannes, dont j'eus moi-même le privilège d'être encore l'élève, et au nom duquel je tiens à associer celui de Sylvain Lévi, le grand indianiste qui se fit aussi sinologue et auquel je dois tant.

Ainsi s'éteint une double génération qui a prouvé avec éclat, dans une discipline faisant appel à l'esprit d'entreprise et de décou verte, toute la vitalité de la science française contemporaine. Grâce à de tels ouvriers, grâce déjà à leurs prédécesseurs, c'est à la France qu'était revenue en Europe la direction des études chinoises; c'est à elle qu'il incombait en quelque sorte, dans les échanges culturels, de représenter l'Europe vis -à-vis de la Chine. Maspero le savait bien, lui qui ne manquait pas une occasion de se référer, pour nous faire pénétrer dans le passé de la Chine, à des analogies tirées de la civilisation méditerranéenne dont nous sommes les héritiers : il obéissait ainsi au dessein bien français de ramener la civilisation chinoise à des dénominateurs communs pour l'intégrer dans l'en semble d'un humanisme universel.

Est-ce l'heure d'interrompre de tels travaux ? Cet humanisme, les événements qui bouleversent l'univers nous en font une nécessité. Quelque audience est due aujourd'hui à ceux qui avaient pres senti la bagarre et dont le regard s'était porté au loin: Mal éveillés d'un cataclysme qui se laissait pourtant prévoir, nous avançons comme à tâtons dans un monde à tel point labouré que tout peut y paraître neuf. Mais le temps n'est pas aboli ; s'affrontant, après la tempête, à la croisée des routes, les nations traînent avec elles leur bagage de traditions. Où donc la Chine aurait-elle puisé la force de résister sans armes au déluge des bombes et de la propagande ennemies, si ce n'est dans cette tradition confucéenne qui lui avait enseigné la suprématie d'une morale gratuite ? Affaissées dans les ruines aux deux bouts du vieux 
continent, l'Europe et la Chine demeurent les gardiennes d'un passé que l'humanité ne sau rait renier sans danger. Plus d'un problème les rapproche ; plus d'une difficulté leur est commune. Il est à souhaiter qu'en leurs épreuves elles trouvent tout au moins l'occasion d'un contact plus étroit, et que la France continue à porter pour l'Europe, en ce qui concerne la Chine, le témoignage de sa science et de son expérience.

Ce sont de telles réflexions qui m'ont enhardi à briguer, tout indigne que je m'en sentisse, la périlleuse responsabilité d'assurer dans cette chaire le relais des études chinoises. J'y fus encouragé par le maître que nous pleurons, Paul Pelliot, ce philologue de génie dont l'érudition avait fini par s'étendre à toutes les branches de l'orientalisme, mais qui était parti de la sinologie et n'a cessé de réserver à la Chine, à son rayonnement extérieur, à l'histoire de ses religions, de ses arts et de ses livres, une place de choix dans son ouvre immense. J'ai cru entendre aussi la voix de Henri Maspero, à la fois bienveillante et stricte, m'e ngageant à ne pas me dérober. L'avenir est obscur, l'héritage est lourd : puissé-je n'y pas faillir et contribuer à entretenir, dans la mesure de mes forces, et à transmettre la flamme qu'ils ont portée si haut !

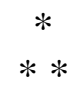


Nom du document : demieville_maspero.doc

Dossier :

C:ICSSIChineWord051204

Modèle :

C:IWINDOWS $\backslash$ Application

DatalMicrosoft $\backslash$ Modèles $\backslash$ Normal.dot

Titre :

Sujet :

Henri Maspero et l' avenir des éudes chinoises

Auteur : série Chine

Mots clés :

Paul Demiéville

bouddhisme, taoisme, histoire de la Chine

Commentaires : http://www.uqac.ca/Classiques_des_sciences_sociales/

Date de création : $\quad$ 02/09/05 22:02

$\mathrm{N}^{\circ}$ de révision : $\quad 14$

Dernier enregistr. le : 07/09/05 22:04

Dernier enregistrement par : Pierre Palpant

Temps total d' édition88 Minutes

Dernière impression sur : $\quad$ 08/09/05 00:22

Tel qu' à la dernière impression

Nombre de pages :

19

Nombre de mots : 7818 (approx.)

Nombre de caractères : 44565 (approx.) 